\title{
Astenopia digital em controladores de tráfego aéreo: diagnóstico e avaliação na Força Aérea Portuguesa
}

\author{
Digital-related eye strain in air traffic control specialists: diagnosis \\ and evaluation in the Portuguese Air Force staff \\ Inês Passos ${ }^{1}$ (D), Sofia Vidigal e Almada² (O), Pedro Reis ${ }^{2}$ (C) \\ Centro Hospitalar Universitário de Lisboa Central, Lisboa, Portugal. \\ 2 Centro de Medicina Aeronáutica da Força Aérea Portuguesa, Lisboa, Portugal.
}

Descritores:

Astenopia; Controladores de tráfego aéreo; Terminais de computador

Keywords: Asthenopia; Air traffic control specialists; Computers

Submetido: 01/03/2021

Aceito: 26/3/2021

Autor correspondente: Inês Passos Centro Hospitalar Universitário de Lisboa
Central Rua José António Serrano, 1150-199 Lisboa, Portugal E-mail: passos_ines@sapo.pt

Instituição: Centro de Medicina Aeronáutica da Força Aérea, Lisboa, Portugal.

Fonte de auxílio à pesquisa: não financiado.

Conflitos de interesse: os autores declaram que não há conflitos de interesses.

\section{RESUMO}

A função de controlador aéreo exige várias horas de visualização de ecrãs, o que torna esses profissionais um grupo particularmente exposto à astenopia digital. Procuramos, com este artigo de revisão, compreender quais os métodos quantitativos e qualitativos usados atualmente para diagnosticar e avaliar a fadiga ocular em controladores de tráfego aéreo. Trata-se de pesquisa bibliográfica utilizando as bases de dados PubMed $₫$ e ClinicalKey ${ }^{\circledR}$, usando palavras-chave, tendo sido selecionados artigos de revisão e estudos observacionais com publicação posterior ao ano 2000, de modo que os termos buscados estivessem no título e/ou resumo do trabalho. Excluímos artigos em línguas que não inglesa. Existem vários métodos para melhor caracterizar a astenopia digital, destacando-se os subjetivos, como questionários, e os objetivos, que procuram, por um lado, diagnosticar o olho seco, bem como alterações da acomodação e da convergência. Da análise efetuada, ressaltou-se uma escassez de estudos prospectivos com Níveis de Evidência moderados e altos relacionados com a aplicação dos vários métodos de diagnóstico. A Computer-Vision Symptom Scale parece ser um questionário confiável e que pode ser aplicado aos controladores de tráfego aéreo, para diagnóstico de astenopia digital. Vários métodos objetivos podem também ser utilizados para essa avaliação, sendo importante, numa fase posterior, aplicar esses dois tipos de métodos de diagnóstico para avaliar a prevalência dessa patologia em controladores de tráfego aéreo.

\section{ABSTRACT}

The work of air traffic control specialists require several hours looking at screens, and they comprise a group particularly exposed to digital-related eye strain. In this review we aim to understand the quantitative and qualitative methods currently used to diagnose and evaluate asthenopia in air traffic control specialists. A bibliographic search was carried out at the databases PubMed ${ }^{\circledR}$ and ClinicalKey ${ }^{\circledR}$ using keywords, and selecting review articles and observational studies, dated after 2000, containing the keywords in the title and/o abstract. Articles not published in English were excluded. There are several subjective and objective methods to better describe digital-related eye strain, including questionnaires, aiming to make diagnosis of dry eye disease or changes in accommodation and convergence. From our analysis, there are few prospective studies with moderate and high levels of evidence regarding these diagnostic methods. The Computer-Vision Symptom Scale seems to be a reliable questionnaire to be applied to air traffic control specialists, for diagnosis of digital-related eye strain. Several objective methods can also be employed in this evaluation, and, in a later stage, it will be important to apply both diagnostic methods to evaluate the prevalence of this condition in air traffic control specialists. 


\section{INTRODUÇÃO}

A fadiga ocular em utilizadores de monitores, ou video display terminals (VDTs), também designada astenopia digital (AD) ou computer vision syndrome, consiste num grupo de sintomas oculares e relacionados com a visão, que advêm do uso prolongado e da consequente exposição a VDTs.

Na Força Aérea Portuguesa, os controladores de tráfego aéreo (em inglês: air traffic controller), designados como Técnicos de Operações de Circulação Aérea e Radar de Tráfego (TOCART), encontram-se sob risco acrescido, pelas particularidades das operações que realizam.

A AD é uma patologia transitória relacionada com a utilização de aparelhos digitais e que resulta do esforço aumentado para a visão de perto, desde o aumento da acomodação e convergência, até a atenção visual e diminuição da frequência do pestanejo que, quando mantido, leva à exaustão dos músculos oculares e à astenopia. ${ }^{(1)}$ Caracteriza-se pelo aparecimento de vários sintomas que podem ser categorizados como oculares, tais como cefaleia, dor ocular, ardor, hiperemia ou sensação de corpo estranho, e como visuais, como visão turva quer para perto, quer para longe. ${ }^{(2)}$ Se é verdade que essa patologia vem crescendo na população em geral, inerente ao uso frequente de aparelhos digitais para fins recreativos, não é menos verdade que, profissionalmente, ela tenha um peso também significativo. Os controladores de tráfego aéreo militares dependem especificamente do uso de VDTs para a realização de suas tarefas. No entanto, não existe, tanto quanto pudemos apurar em nossa pesquisa, informação relativamente à prevalência dessa patologia nessa especialidade da Força Aérea, o que torna esta revisão particularmente relevante, no sentido de apurar as formas de detecção da AD nos controladores de tráfego aéreo e reformular ou manter procedimentos de mitigação dessa patologia.

\section{MÉTODOS}

Para a realização desta revisão bibliográfica, foram pesquisados artigos nas bases de dados PubMed ${ }^{\circledR}$ e ClinicalKey ${ }^{\circledR}$ em língua inglesa. Foram utilizadas as palavras-chave: "computer vision syndrome", "computer vision syndrome and questionnaire", "digital asthenopia", "eye-strain", "computer vision syndrome and video display terminals", "computer vision syndrome and monitors" e "computer vision syndrome and dry eye disease". Utilizado em conjunto com os anteriores, o termo air traffic controller resultou em apenas três artigos relacionados indiretamente com o tema, o que revelou a escassez de informação relativamente ao assunto. Tivemos como critérios de inclusão a seleção de artigos posteriores ao ano de 2000, cujo título e resumo estivessem relacionados com a revisão em causa, que fossem artigos de revisão ou estudos observacionais. Foram excluídos artigos em línguas diferentes da língua inglesa e que não cumprissem os critérios de inclusão.

\section{RESULTADOS Sintomatologia}

Na literatura consultada, a AD teve prevalência que variou entre 50\% e 90\% dos utilizadores de monitores. ${ }^{(2,3)}$ Esse valor é variável, uma vez que muitos estudos avaliaram diferentes períodos de exposição, sendo que, na maioria, com o aumento do período de exposição, aumentou também a prevalência de sintomatologia ocular. ${ }^{(4)}$

Essa patologia cursa com uma constelação de sintomas próprios, que podem ser agrupados em duas categorias: ${ }^{(3)}$ associados ao estress acomodativo e da visão binocular, como cefaleias, dor ocular, eye strain ou visão turva, e os sintomas externos associados ao olho seco, como ardor ou lacrimejo.

A visão turva, quer para perto, quer para longe, é sintoma frequentemente associado à utilização prolongada de computadores, podendo resultar de resposta acomodativa deficiente ou da dificuldade do relaxamento completo da mesma resposta acomodativa após tarefas para perto. ${ }^{(5)} \mathrm{O}$ esforço para perto pode também ser um fator causal para o desenvolvimento de um shift miópico. ${ }^{(6)}$

Já a sintomatologia relacionada com o olho seco pode ser desencadeada por vários fatores, dos quais se destacam: taxa de pestanejo reduzida, exposição aumentada da superfície ocular ao dirigir o olhar superiormente, fatores ambientais (como ar condicionado) ou mesmo presença de outras doenças (sistêmicas ou oculares).

Está plenamente estudado o impacto da síndrome do olho seco na qualidade de vida dos doentes. O Nível de Evidência é alto para seu impacto negativo mensurável em várias atividades do dia a dia, nomeadamente na utilização de VDTs. ${ }^{(7)}$

Na Força Aérea, a especialidade de controlador de tráfego aéreo implica na realização de operações de controle em que a exposição a VDTs é constante. Não obstante existirem normas europeias que regulam a profissão civil e procuram minimizar os efeitos da fadiga (2 horas de posição de controle seguidas de 10 minutos de descanso e mais 10 minutos por cada hora de trabalho - Eurocontrol), ${ }^{(8)}$ na Força Aérea não existem ainda protocolos que definam essa situação, tornado extremamente relevante a avaliação dos fatores desencadeantes. 
Existem vários fatores que parecem influenciar nas queixas visuais dos doentes. Estudo cross-sectional ${ }^{(9)}$ chegou à conclusão de que 53\% dos utilizadores de computadores referem eye strain, 42,66\% cefaleia, 34\% diminuição da visão para perto e $32 \%$ lacrimejo, sugerindo que a sintomatologia é mais frequente após 6 horas de exposição. O mesmo estudo demonstrou que as queixas oculares são mais frequentes em utilizadores que não usam óculos, bem como há maior incidência dessa sintomatologia em utilizadores que não mantinham os computadores a uma distância adequada. Estudo controlado e randomizado com 44 participantes ${ }^{(10)}$ procurou comparar a presença de sintomatologia ocular após a leitura de um documento em iPAD ou documentos impressos. Foram verificados níveis significativamente mais altos de eye strain e irritação ocular no grupo que usou o iPAD face ao grupo controle. Este estudo corrobora a teoria de que, mais importante que o trabalho para perto como fator de sintomatologia ocular, a utilização de ecrãs parece ser o fator-chave.

Também parece ser relevante o tipo de monitor utilizado, uma vez que monitores do tipo cathode-ray tube (CRT) estão habitualmente associados à maior prevalência de fadiga ocular que monitores liquid-crystal display (LCD). (4)

Na Força Aérea, os monitores disponíveis para os controladores de tráfego aéreo são de tipo LCD sintetizados por computador, não obrigando à ausência de luminosidade, o que beneficia os utilizadores. Lin et al. ${ }^{(11)}$ procuraram estudar o efeito do glare no desenvolvimento de fadiga ocular, expondo os participantes a três tipos diferentes de displays (glossy, matte e glare-free), quer em ambiente com fonte de luminosidade causadora de glare, que em ambiente sem ela. Monitores glare-free puderam aliviar a fadiga ocular e preservar o desempenho visual, mas também a luminosidade uniforme pode diminuir a variação do diâmetro pupilar, prevenindo o eye strain.

Na atualidade, muito se têm debatido os efeitos oculares potencialmente nefastos da luz azul e as formas como minimizar essa exposição. Um estudo original de pequena dimensão ${ }^{(2)}$ procurou avaliar o efeito de um filtro para a luz azul nos sintomas de fadiga ocular após leitura num monitor. Mesmo eliminando cerca de 99\% da luz azul emitida através do filtro, não existia eficácia na redução de sintomas de olho seco comparativamente a um filtro de densidade neutra.

É também relevante observar que o trabalho constante com aparelhos eletrônicos implica no controle de temperatura por meio de ar condicionado nas salas de controle, procurando manter uma temperatura ambiente, na ordem dos $22^{\circ} \mathrm{C}$. A baixa umidade do ar condicionado, quando inferior a 40\%, associada a correntes de ar e a temperaturas elevadas, ${ }^{(13)}$ parece ser fator importante e predisponente para eventual sintomatologia de olho seco por disrupção evaporativa do filme lacrimal, levando a hiperosmolaridade e ao desconforto ocular. Nos controladores de tráfego aéreo, dois desses fatores são verificados no local de trabalho, podendo ser importantes no desenvolvimento dessa patologia.

\section{Métodos de diagóstico}

Para avaliar a sintomatologia em utilizadores de VDTs, podemos utilizar métodos subjetivos e objetivos. ${ }^{(3)}$

Nos métodos subjetivos, podemos incluir vários tipos de questionários que fazem uma avaliação qualitativa dessa patologia. O questionário Ocular Surface Disease Index (OSDI), por exemplo, avalia queixas relacionadas com olho seco. O Portuguese Group of Ergophthalmology Questionnaire (PGEQ) consiste em quatro questões que o doente classifica de zero a quatro pontos (equivalentes a nunca ou a todos os dias). ${ }^{(1)}$

O Computer Vision Syndrome Questionnaire $\left(\right.$ CVS- $\left.Q^{\circledR}\right)$, que revelou propriedades psicométricas aceitáveis para o controle da saúde visual em utilizadores de monitores, seleciona 16 sintomas, quantificados conforme a frequência com que ocorrem, numa escala de zero a três (de nunca a quase diariamente), e a intensidade de cada um, também numa escala de zero a três (de moderado a muito intenso). ${ }^{(14)} \mathrm{O}$ Computer-Vision Symptom Scale $\left(\right.$ CVSS17 $^{(15)}$ desenvolvido em espanhol como forma de quantificar a sintomatologia ocular ligada ao uso de computadores demonstrou boa fiabilidade e consistência interna. Apresenta 17 itens, permitindo explorar 15 diferentes sintomas, e surge, assim, como uma ferramenta útil para quantificar a $\mathrm{AD}$, existindo em formato on-line disponível a qualquer utilizador.

Existem também vários métodos objetivos que permitem identificar alterações nos utilizadores de monitores e podem ser uma forma de rastreio de AD. Esses testes procuram avaliar, por um lado, a presença de olho seco e alterações da superfície ocular e, por outro, alterações da acomodação e convergência.

O teste de Schirmer sem anestesia, o tempo de rotura lacrimal ou o interferômetro (DR-1 $\left.{ }^{\mathrm{a}}{ }^{\mathrm{TM}}\right)$ foram alguns dos métodos usados por um estudo realizado pelo Grupo Português de Ergoftalmologia, ${ }^{(1)}$ que procurou avaliar a prevalência de manifestações de olho seco e fadiga ocular numa população de 77 indivíduos. Apresentam-se como testes seguros, que permitem diagnosticar essa patologia. Estudo prospectivo ${ }^{(16)}$ 
mostrou que o teste de osmolaridade normal em doentes com sintomas de olho seco apresenta-se como uma boa ferramenta para diferenciar essa patologia de outras doenças da superfície ocular, utilizando como valor normal <308mOsm/L. O mesmo estudo português ${ }^{(1)}$ procura avaliar a presença de fadiga ocular identificando o ponto próximo de acomodação e convergência, utilizando a régua de Força Aérea Real (RAF). Há também um artigo de revisão sobre a presença de espécies reativas de oxigênio na superfície ocular e sua implicação na inflamação ocular e olho seco. ${ }^{(17)}$

\section{CONCLUSÃO}

A revisão bibliográfica mostra que há poucos estudos direcionados para essa população específica. A maioria dos trabalhos publicados utiliza métodos subjetivos para caracterizar essa patologia. A utilização de métodos objetivos de avaliação das alterações da superfície ocular e da composição da lágrima pode elucidar aspetos fisiopatológicos relacionados com a astenopia digital.

Seria também importante o estabelecimento de protocolos de segurança para a prevenção da astenopia digital nos controladores de tráfego aéreo. Intervindo nas várias causas, é relativamente fácil adaptar a realidade atual e implementar medidas simples, que minimizem o eventual impacto dessa patologia. Devem ser adoptadas medidas de descanso ocular periódicas, assim como o posicionamento do ecrã deve ser ajustado, utilizando uma distância de $35 \mathrm{~cm}$ a $40 \mathrm{~cm}$, com ângulo de cerca de $10^{\circ} \mathrm{a}$ $20^{\circ}$, para ajudar a prevenir a astenopia digital. Em controladores de tráfego aéreo com queixas de olho seco, a utilização de lubricantes oculares pode ser um importante contributo para minimizar os sintomas.

\section{REFERÊNCIAS}

1. Vaz FT, Henriques SP, Silva DS, Roque J, Lopes AS, Mota M. Digital Asthenopia: Portuguese Group of Ergophthalmology Survey. Acta Med Port. 2019;32(4):260-5.

2. Gowrisankaran S, Sheedy JE. Computer vision syndrome: A review. Work. 2015;52(2):303-14.

3. Sheppard AL, Wolffsohn JS. Digital eye strain: prevalence, measurement and amelioration. BMJ Open Ophthalmol. 2018;3(1):e000146.

4. Agarwal S, Goel D, Sharma A. Evaluation of the Factors which Contribute to the Ocular Complaints in Computer Users. J Clin Diagn Res. 2013;7(2):331-5.

5. Rosenfield M. Computer vision syndrome: a review of ocular causes and potential treatments. Ophthalmic Physiol Opt. 2011;31(5):502-15.

6. Blehm C, Vishnu S, Khattak A, Mitra S, Yee RW. Computer vision syndrome: a review. Surv Ophthalmol. 2005;50(3):253-62

7. Miljanović B, Dana R, Sullivan DA, Schaumberg DA. Impact of dry eye syndrome on vision-related quality of life. Am J Ophthalmol. 2007;143(3):409-15

8. European Organization for the Safety of Air Navigation, Managing Shiftwork in European ATM: Literature Review;2006.

9. Agarwal S, Goel D, Sharma A. Evaluation of the factors which contribute to the ocular complaints in computer users. J Clin Diagn Res. 2013;7(2):331-5.

10. Maducdoc MM, Haider A, Nalbandian A, Youm JH, Morgan PV, Crow RW. Visual consequences of electronic reader use: a pilot study. Int Ophthalmol. 2017;37(2):433-9.

11. Lin CW, Yeh FM, Wu BW, Yang $\mathrm{CH}$. The effects of reflected glare and visual field lighting on computer vision syndrome. Clin Exp Optom. 2019;102(5):513-20

12. Palavets T, Rosenfield M. Blue-blocking filters and digital eyestrain. Optom Vis Sci. 2019;96(1):48-54.

13. Parihar JK, Jain VK, Chaturvedi P, Kaushik J, Jain G, Parihar AK. Computer and visual display terminals (VDT) vision syndrome (CVDTS). Med J Armed Forces India. 2016 Jul;72(3):270-6

14. Seguí Mdel M, Cabrero-García J, Crespo A, Verdú J, Ronda E. A reliable and valid questionnaire was developed to measure computer vision syndrome at the workplace. J Clin Epidemiol. 2015;68(6):662-73.

15. González-Pérez M, Susi R, Antona B, Barrio A, González E. The ComputerVision Symptom Scale (CVSS17): development and initial validation. Invest Ophthalmol Vis Sci. 2014;55(7):4504-11.

16. Brissette AR, Drinkwater OJ, Bohm KJ, Starr CE. The utility of a normal tear osmolarity test in patients presenting with dry eye disease like symptoms: A prospective analysis. Cont Lens Anterior Eye. 2019;42(2):185-9.

17. Dogru M, Kojima T, Simsek C, Tsubota K. Potential role of oxidative stress in ocular surface inflammation and dry eye disease. Invest Ophthalmol Vis Sci. 2018;59(14):DES163-DES168. 